\title{
The Regulation of Direct-to-Consumer Advertising of Pharmaceuticals in a Managed Care Setting
}

\author{
July 10, 2014
}

\begin{abstract}
We analyse direct-to-consumer advertising (DTCA) in the prescription drug market, when a regulator imposes a fine for misleading advertisements (truth-in-advertising regulation) and doctors face pressure to contain prescribing costs. The efficacy of a drug is based on scientific evidence as well as on patient-specific characteristics. Patients do not possess information on either dimension of efficacy. Pharmaceutical firms observe the scientific data and use DTCA to convey this information to patients. Doctors observe both the scientific data and patient-specific characteristics, and provide treatment recommendations. We develop a model in which DTCA is followed by a doctor-patient signalling game. We show that truth-in-advertising regulation increases the credibility of DTCA and may increase both doctor-patient conflict and prescriptions for an expensive new drug - a market stealing effect. Tighter regulation may encourage more DTCA, and may even encourage more false advertising.
\end{abstract}

\section{Introduction}

The marketing of ethical medicines is a highly regulated activity. In the U.S. direct-to-consumer advertising (DTCA) must restrict statements to verifiable 
facts and only promote the use of the drug for approved indications (Danzon and Keuffel, 2014). However, DTCA remains controversial, and episodes such as the withdrawal of Vioxx have amplified calls for tightening of the regulation of DTCA. Vioxx was amongst the most heavily advertised products in the market (Bradford et al., 2006) but was later found to have fatal side-effects leading to large-scale mortality (Vaithianathan et al., 2009). Its rapid take-up was partly blamed on intensive marketing campaigns, prompting calls for tighter regulations and better enforcement of truth-in-advertising rules (U.S. General Accounting Office, 2002; Donohue, Cevasco and Rosenthal, 2007; Shuchman, 2007).

An open question is whether increased regulation or monitoring will discipline advertisers as intended. Nelson (1974) pointed out that truth-inadvertising regulation may have the perverse effect of encouraging more advertising, since regulation enhances the credibility - and therefore the value - of advertisements. Sauer and Leffler (1990) found some empirical support for Nelson's hypothesis. However, Nelson does not consider markets with "learned intermediaries" who might contradict the advertised message.

The present paper develops a signalling model in which a patient consults a physician about whether to take a new drug after observing DTCA. The patient's treatment decision is based on what they infer from DTCA as well as the doctor's recommendation. Drug advertising is randomly audited for truthfulness.

We assume that physicians face pressure to limit prescribing of expensive new drugs - as in a managed care environment - so physicians are imperfect agents of their patients. Nevertheless, in the absence of DTCA, patients comply with their physicians' suggested treatments. Advertising changes equilibrium behaviour in the physician-patient consultation by encouraging the patient to challenge a physician who recommends against the advertised drug. In the model, DTCA distorts the physician's recommendation towards the advertised drug and also leads to greater levels of conflict with patients.

Our model therefore illustrates one possible mechanism by which DTCA might facilitate market stealing - shifting demand to the advertised brand. It 
is also consistent with empirical evidence on the detrimental effects of DTCA on patient-physician trust, particularly in a managed care or HMO setting (Kravitz et al., 2005; Spence et al., 2005).

We examine two types of market-stealing equilibria in detail: one in which all advertising is truthful, and another in which some advertising is false. Although we do not characterise all equilibria of the model, our results are nevertheless general in the following sense: if DTCA is used in equilibrium, ${ }^{1}$ then the level of prescribing of the advertised drug will be higher than under a scenario in which DTCA is banned.

There have been a number of empirical studies of the effects of DTCA on prescribing (e.g., Berndt et al., 1995, 1997; Calfee, Winston and Stempski, 2002; Iizuka, 2004; Iizuka and Jin, 2005; Ling, Berndt and Kyle, 2002; Rosenthal et al., 2003). The evidence suggests that DTCA can be effective in increasing own-brand demand. Narayanan, Desiraju and Chintagunta (2004) and Kalyanaram $(2008,2009)$ find that DTCA increased the market share of the advertised drug. Wosinska (2002) also finds a market stealing effect of DTCA, but only when the drug is subsidised for the patient.

There is also evidence that this market-stealing effect might work by encouraging patients to request the advertised medicine. Liu and Gupta (2011) analyse prescribing data for patients newly diagnosed with hyperlipidemia, and find that DTCA has a positive and statistically significant effect on patient requests for the advertised brand. In Kravitz et al. (2005) actors were randomly assigned to make 298 unannounced visits to physicians. They found that $37 \%$ of patients requesting a named brand received a prescription for the drug, compared to $10 \%$ of patients who made a general drug request and none of the patients who did not request any drugs. They conclude that as long as DTCA can persuade patients to mention a brand, physicians can be induced to change their prescribing decisions.

Previous theoretical analyses of DTCA have tended to focus on market expansion effects, rather than market stealing.

\footnotetext{
${ }^{1}$ More precisely still, in any restricted equilibrium - as defined in the Supplementary Material.
} 
Brekke and Kuhn (2006) analyse the interaction between DTCA, price setting and detailing (marketing to physicians). They model DTCA as directly informative: consumers do not question the truthfulness of advertised messages, and are prompted to visit their physicians by learning the advertised information. By contrast, we assume that patients visit their physicians whether or not they see DTCA, but our patients do not unquestioningly believe the content of DTCA. Our model explains why DTCA may persuade consumers to switch drugs, despite their rational incredulity and the intermediation by physicians. In Brekke and Kuhn, it is detailing that drives the prescribing decision, which is made by the physician. There is no detailing in our model (though this an obvious avenue for further research), and patients have sovereignty over the prescribing decision. The exercise of this sovereignty is limited, however, by the need to pay a "conflict cost" to reject the physician's recommendation.

Rubin and Schrag (1999) likewise treat DTCA as directly informative. Intensity of advertising increases brand awareness, which in turn encourages the HMO to place the drug on its formulary. Rubin and Schrag show that tighter regulation, which imposes higher compliance costs on the advertiser, can have ambiguous effects if DTCA is a strategic complement to price. With higher-cost advertising, the drug company advertises less and charges a lower price. This reduces awareness amongst consumers but the lower price increases consumption amongst the brand-aware consumers.

Neither of these papers addresses the credibility of DTCA or the regulation of content.

We explore the effect of truth-in-advertising regulation - the frequency of auditing and the penalties for false advertising - on the incentive to advertise. Stricter regulation may increase the credibility of DTCA and may therefore lead to increased DTCA and more physician-patient conflict. Stricter regulation may even help to support false advertising. Our paper therefore challenges the presumption that tighter, or better enforced, truth-in-advertising rules are effective mechanisms for reducing the harmful effects of DTCA.

A critical assumption of our model is that physicians are imperfect agents 
of their patients. Physicians employed by an HMO or managed care organisation may face cost-containment mechanisms such as utilisation reviews, capitation of provider payments and selective contracting with providers, all of which challenge the physician's autonomy and reduce the emphasis on maximising health gain. They may therefore be less inclined to prescribe expensive new drugs than their patients would like. ${ }^{2}$ Domino and Salkever (2003), for example, find that physicians in an HMO setting are less likely to prescribe expensive anti-depressants. Wynia et al. (2003) found that a substantial proportion of doctors covered by managed care admitted to not mentioning effective services to patients when these services were not covered.

There is also evidence that patients are well aware of the pressures under which doctors operate. Schlesinger (2002) reports the results of interviews with a sample $(n=1,527)$ of randomly selected patients about their faith in the medical profession. Half of the respondents agreed with the statement that "doctors no longer place your well-being above concerns about health care costs". Kao et al. (1998) surveyed 2,086 enrollees in a large insurer who were distributed across fee-for-service and a variety of managed care plans. They found that patients under the managed care schemes were statistically significantly less likely to trust their physicians to put their health ahead of costs.

Patients in managed care schemes therefore face two biased sources of information: the producers of expensive new drugs and their own physicians. This complicates the patient's treatment decision, but creates an opportunity for drug producers to offset managed care pressure against prescribing their

\footnotetext{
${ }^{2}$ Under the Medicaid "fail first" regime, doctors are required to try a cheaper drug first, while some drugs require the doctor to complete paper work and obtain prior approval to prescribe. With the advent of Medicare Part D, Medicare beneficiaries can also enrol in HMO-type arrangements which include prescription drugs. The share of drugs with utilisation management requirements amongst these type of plans increased from $18 \%$ in 2007 to $28 \%$ in 2011, with prior authorisation being the most pervasive management strategy. Prior authorisation imposes costs on doctors (Epling, Mader and Morley, 2014) and encourages them to switch their prescribing to drugs for which prior authorisation is not needed (Law et al., 2010).
} 
products. Because the drug producer's bias is in the opposite direction to that of the physician, there is scope for DTCA to be effective in persuading the patient to insist on the new drug, even if this means going against his physician's advice.

\section{The model}

A population of patients is being treated for a chronic condition and regularly visit their physicians. ${ }^{3}$ Patients have a common von Neumann-Morgenstern (vNM) utility function which depends on health, the level of any co-payments and the level of conflict with the doctor. ${ }^{4}$ Each patient is currently being treated with generic $\operatorname{drug} \alpha$, but a new, patented medication $\beta$ has just been introduced to the market.

All agents, including patients, are aware of the existence of $\beta$ and the conditions that it is designed to treat ${ }^{5}$ but are uninformed about its efficacy. The latter is determined by:

(i) The average quality of $\beta$, as revealed by the randomized controlled trial (RCT) data. This is known to both the pharmaceutical firm and the physician.

(ii) Patient-specific factors which determine the quality of the match between the patient and the drug. These are ascertained by a medical examination, so are known only to the physician.

For a given patient, the efficacy of $\operatorname{drug} \beta$ is indexed by the state $s \in$ $S=[\underline{s}, 1]$, where $\underline{s}<0$. The state determines the additional health gain from taking $\beta$ rather than $\alpha$ (net of any co-payment for drug $\beta$ ), expressed

\footnotetext{
${ }^{3}$ According to the U.S. General Accounting Office (2002), all of the 15 most heavily advertised drugs in 2000 were treatments for chronic conditions.

${ }^{4} \mathrm{~A}$ precise specification of utility is given below.

${ }^{5}$ We exclude brand awareness as a motivation for DTCA. This is often established through media campaigns (Cassels et al., 2003).
} 
in vNM utils. The state is unobserved by the patient, but is known to the physician at the time of consultation.

\section{The consultation game}

We first describe a physician-patient consultation game in which the patient visits the physician for a drug recommendation.

At the time of consultation, the patient has prior beliefs over $S$ described by the distribution function $F$. Later we introduce advertising by pharmaceutical firms, in which case $F$ will incorporate anything the patient has inferred about drug quality from DTCA. For now, though, we take $F$ as given and explore what happens during the patient-doctor consultation.

We model this consultation process as a signalling game. Nature selects the state $s \in S$ according to the distribution $F$. The physician ("she") observes the state and makes a recommendation $r \in\{\hat{\alpha}, \hat{\beta}\}$. Here, $r=\hat{\alpha}$ denotes a recommendation of $\operatorname{drug} \alpha$ and $r=\hat{\beta}$ a recommendation of drug $\beta$. The patient ("he") observes the physician's recommendation, but not the state, and chooses a treatment $t \in\{\alpha, \beta\}$.

The doctor's strategy is a mapping $\sigma^{d}: S \rightarrow[0,1]$, where $\sigma^{d}(s)$ is the probability that $r=\hat{\beta}$ in state $s$. The patient's strategy is a mapping $\sigma^{p}:\{\hat{\alpha}, \hat{\beta}\} \rightarrow[0,1]$, where $\sigma^{p}(r)$ is the probability that $t=\beta$ given recommendation $r$. Thus, strategies indicate the probability of recommending/choosing drug $\beta$.

A conflict occurs whenever the patient's treatment choice is different from the physician's recommendation. The doctor and patient suffer some disutility from conflict $\left(c_{d}>0\right.$ and $c_{p}>0$, respectively).

The patient's vNM utility payoff is

$$
u^{p}(r, t, s)=\left\{\begin{array}{cc}
v-\mathbb{I}[r=\hat{\beta}] c_{p} & \text { if } t=\alpha \\
v+s-\mathbb{I}[r=\hat{\alpha}] c_{p} & \text { if } t=\beta
\end{array}\right.
$$

where $\mathbb{I}[\cdot]$ denotes the indicator function and $v$ is the treatment benefit from $\operatorname{drug} \alpha$. Since payoffs are expressed in vNM utils, our model imposes no 
particular risk attitude on patients. ${ }^{6}$

The doctor's vNM utility payoff is :

$$
u^{d}(r, t, s)=\left\{\begin{array}{cc}
v-\mathbb{I}[r=\hat{\beta}] c_{d} & \text { if } t=\alpha \\
v+(s-\tau)-\mathbb{I}[r=\hat{\alpha}] c_{d} & \text { if } t=\beta
\end{array}\right.
$$

The utility penalty $\tau \in(0,1)$, which is imposed when $\beta$ is prescribed $(t=\beta)$, embodies the doctor's bias against $\beta$. Apart from this bias, the physician's utility function reflects altruistic preferences (or a competitive pressure to maximise patient utility).

With these payoffs, the physician is an imperfect agent of the patient. When $s \in(0, \tau)$ the patient and physician disagree about the preferred treatment: the patient would like $\beta$ but the physician prefers the cheaper alternative, $\alpha$. We assume that the patient understands the doctor's incentives; in particular, patients know the value of $\tau .^{7}$

Since none of our results depends on the value of $v$, we set $v=0$ henceforth. ${ }^{8}$ We also make the following:

\footnotetext{
${ }^{6}$ To clarify, imagine a patient who derives vNM utility $u(H, M)$ from his level of health $H$ and his monetary wealth $M$. This function embodies the patient's attitude to risk in these two dimensions. We impose no restrictions on its curvature. Let $\Gamma$ denote a "fundamental" state space that summarises the relative health benefit from taking $\beta$ rather than $\alpha$. Then $H$ is a function of $(\gamma, t)$, where $\gamma \in \Gamma$ is the state and $t \in\{\alpha, \beta\}$ is the treatment; while $M$ is a function of $t$. Moreover, $H(\gamma, \alpha)$ is constant in $\gamma$. Let $v=u(H(\gamma, \alpha), M(\alpha))$ be the vNM utility from treatment $\alpha$. We may therefore transform the "fundamental" state space $\Gamma$ into a our state space $S$ by defining:

$$
s(\gamma)=u(H(\gamma, \beta), M(\beta))-v
$$

In other words, $u(H(\gamma, \beta), M(\beta))=v+s(\gamma)$. We therefore define the patient's vNM utility as $v+s(\gamma)$ less any conflict cost (and drop the redundant reference to $\gamma$ ). The patient's risk attitude is incorporated in the change of variable: the transformation of $\gamma$ into $s$. This implies that the patient's prior distribution $(F)$ over $S$ will be affected by his risk attitude. However, once $S$ and $F$ are specified, risk attitude plays no further role in the analysis.

${ }^{7}$ See the Introduction for a discussion of this assumption.

${ }^{8}$ Since $S$ has an upper bound normalised to 1 , setting $v=0$ exhausts the allowable normalisations of vNM utility.
} 
Assumption 1 The distribution $F$ is continuous with support $[\omega, 1]$ for some $\omega \in[\underline{s}, 0)$. It is strictly increasing on its support and satisfies $F(\omega)=0$.

By an "equilibrium" of the consultation game, we shall mean a Perfect Bayesian Equilibrium (PBE) that satisfies the Intuitive Criterion (IC). ${ }^{9}$ To simplify matters - at least initially - we focus on equilibria which are responsive and state monotonic:

Definition 1 An equilibrium is responsive if the patient responds differently to each recommendation: $\sigma^{p}(\hat{\alpha}) \neq \sigma^{p}(\hat{\beta})$. Otherwise, the equilibrium is non-responsive.

Definition 2 An equilibrium is state monotonic if $\sigma^{d}(s)$ is weakly increasing in s. That is, if the probability of the doctor recommending drug $\beta$ is weakly increasing in $s$.

In a non-responsive equilibrium, the patient ignores the doctor's recommendation. One such equilibrium - a Beta equilibrium - is discussed below. However, we shall set aside the non-responsive equilibria for now.

There is something obviously counter-intuitive about equilibria that violate state monotonicity, though these too may exist. Proposition 2 implicitly acknowledges them, but we do not give any explicit consideration to such equilibria.

The following simple observation is useful for the analysis of responsive equilibria: ${ }^{10}$

Lemma 1 Suppose $r, r^{\prime} \in\{\hat{\alpha}, \hat{\beta}\}, r \neq r^{\prime}$, and the patient's strategy satisfies $\sigma^{p}\left(r^{\prime}\right)>\sigma^{p}(r)$. Then there exists $s^{*} \in S$ such that the physician's unique best response is $r$ when $s<s^{*}$ and $r^{\prime}$ when $s>s^{*}$. If $s^{*} \in(\underline{s}, 1)$ the physician is indifferent about her recommendation in state $s^{*}$.

\footnotetext{
${ }^{9}$ Because of conflict costs, the consultation signalling game is not of the "cheap talk" variety, so the IC has bite in disciplining off-equilibrium beliefs.

${ }^{10}$ All proofs are in the Appendix.
} 
Suppose $\sigma^{p}\left(r^{\prime}\right)>\sigma^{p}(r)$, and let $s^{*}$ be defined as in Lemma 1. If $\sigma^{p}$ is part of a state monotonic equilibrium then we must have $r^{\prime}=\hat{\beta}$ and $r=\hat{\alpha}$. In such an equilibrium, the doctor recommends drug $\beta$ in states above $s^{*}$ and recommends drug $\alpha$ in states below $s^{*}$. If $s^{*} \in(\omega, 1)$ then both recommendations occur with strictly positive probability in equilibrium (Assumption 1). In this case, $\sigma^{p}(\hat{\beta})$ is an optimal response given posterior beliefs that condition $F$ on $\left[s^{*}, 1\right]$, and $\sigma^{p}(\hat{\alpha})$ is optimal for posterior beliefs that condition $F$ on $\left[\underline{s}, s^{*}\right]$. In particular, $\sigma^{d}\left(s^{*}\right)$ is irrelevant to the patient's optimal response, since $s^{*}$ occurs with probability zero conditional on either recommendation.

It turns out that there always exists such an equilibrium, and it is essentially unique (in a sense made precise in Proposition 1). The nature of the equilibrium depends on whether $\tau$ lies above or below the following parameter:

$$
\bar{s}=\sup \left\{\begin{array}{l|l}
s^{\prime} \in S \mid \int_{\underline{s}}^{s^{\prime}}\left(s-c_{p}\right) d F(s) \leq 0
\end{array}\right\}
$$

Note that $\bar{s}>0$ by Assumption 1. The following lemma clarifies the role of parameter $\bar{s}$.

Lemma 2 Given $s^{*} \in(\omega, 1)$, the condition

$$
\frac{1}{F\left(s^{*}\right)} \int_{\underline{s}}^{s^{*}} s d F(s) \leq c_{p}
$$

is equivalent to $s^{*} \leq \bar{s}$.

Condition (2) is necessary and sufficient for the patient to be willing to accept recommendation $\hat{\alpha}$ when his posterior beliefs condition $F$ on $\left[\underline{s}, s^{*}\right]$. If $\tau \leq \bar{s}$ the doctor can therefore safely recommend her preferred treatment in every state. ${ }^{11}$ In particular, if recommendation $\hat{\alpha}$ reveals to the patient that the state lies in $[\underline{s}, \tau]$, he is happy to choose $t=\alpha$. (He will certainly choose $t=\beta$ when recommendation $\hat{\beta}$ informs him that the state lies in $[\tau, 1]$.) On the other hand, if recommendation $r=\hat{\alpha}$ informs the patient that $s \in[\underline{s}, \tau]$

\footnotetext{
${ }^{11}$ A similar result is obtained by Lanzi and Mathis (2007, Proposition 2).
} 
but $\tau>\bar{s}$, the patient will reject the recommendation. In this case, the physician cannot hope to get her own way in all states.

Proposition 1 There always exists a responsive and state monontonic equilibrium with $s^{*}$ (defined in Lemma 1) lying in $(\omega, 1)$. If $\tau \leq \bar{s}$, then in any such equilibrium:

$$
s^{*}=\tau, \quad \sigma^{p}(\hat{\alpha})=0 \quad \text { and } \sigma^{p}(\hat{\beta})=1
$$

If $\tau>\bar{s}$, then in any such equilibrium:

$$
s^{*}=\bar{s}, \quad \sigma^{p}(\hat{\alpha})=\frac{(\tau-\bar{s})}{(\tau-\bar{s})+c_{d}} \text { and } \sigma^{p}(\hat{\beta})=1
$$

We call an equilibrium satisfying (3) a physician knows best equilibrium $(P K B E)$. In any $\mathrm{PKBE}$, the physician recommends her preferred treatment in every state and the patient accepts either recommendation.

An equilibrium satisfying (4) is called a conflict equilibrium (CE). In such an equilibrium, the physician refrains from recommending drug $\alpha$ in states above $\bar{s}$, which is less than $\tau$. Even so, the patient rejects $r=\hat{\alpha}$ with strictly positive probability: $\sigma^{p}(\hat{\alpha})>0$ when $\tau>\bar{s}$.

Proposition 1 says that the consultation game always possesses a PKBE or a $\mathrm{CE}$, and that the value of $\bar{s}$ determines which equilibrium exists. We will focus on these equilibria in what follows.

In the next section we argue that the producer of $\operatorname{drug} \beta$ may be able to use DTCA to shift $\bar{s}$. It can do so if its advertising alters the distribution $F$. Note that $F$ reflects the patient's beliefs about the net benefit from drug $\beta$, and may be amenable to DTCA. ${ }^{12}$

If advertising is successful in shifting $\bar{s}$ from a value above $\tau$ to a value below $\tau$, the nature of the consultation game changes: from one with a PKBE

\footnotetext{
${ }^{12}$ The true distribution may differ from the patient's perceived distribution. This true distribution is known to the physician (and to the drug firm) but since the physician observes $s$, it is the patient's perceived distribution that is relevant for the consultation game.
} 
(but no $\mathrm{CE}$ ) to one with a $\mathrm{CE}$ (but no PKBE). This change may therefore increase the set of states in which $\beta$ is recommended, as well as ensuring that $\beta$ is prescribed with positive probability in the remaining states. This is a market stealing effect, and will raise the drug firm's profit. ${ }^{13}$

Note that a downward shift in $\bar{s}$ implies that the patient becomes more optimistic about the net benefit of drug $\beta$ : more states must be excluded from the top end of the support of patient beliefs to maintain indifference about accepting or rejecting $r=\hat{\alpha}$. Effective DTCA must therefore persuade the consumer that drug quality is higher than his prior belief. Section 4 shows that DTCA may be persuasive in equilibrium.

The following two subsections discuss alternative equilibria of the consultation game, and the possibility of purifying the CE to obviate the need for patient randomisation. They may be skipped without loss of continuity.

\subsection{Alternative equilibria}

The consultation game may (indeed, will) possess other equilibria, besides the PKBE or CE. Is it reasonable to focus on just these two?

When $\tau \leq \bar{s}$ the PKBE has a compelling logic. As noted previously, if $\tau \leq \bar{s}$ the physician can recommend her preferred drug in every state and be confident that the patient will be willing to accept the recommendation without argument. It seems natural to assume that she will do so.

We can also show:

\section{Proposition 2 If}

$$
-c_{p}<\frac{1}{F(\tau)} \int_{\underline{s}}^{\tau} s d F(s) \leq 0
$$

then a PKBE exists and Pareto dominates any other equilibrium.

\footnotetext{
${ }^{13}$ Whatever the true distribution over $S$, shifting patient perceptions $(F)$ such that the equilibrium switches from a PKBE to a $\mathrm{CE}$ will necessarily raise expected profit, as it (weakly) increases the probability of a $\beta$ prescription in every state.
} 
Condition (5) implies $\tau \leq \bar{s}$ and hence the existence of a PKBE. Under the stronger condition (5), when Pareto dominance is used as an equilibrium selection criterion, it selects the PKBE.

The Supplementary Material has further discussion of equilibria, besides those of the PKBE form, that exist when $\tau \leq \bar{s}$ but (5) does not hold.

The Supplementary Material also considers alternative equilibria (to the CE) when $\tau>\bar{s}$. It is shown that the only other state monotonic equilibria are ones in which $\beta$ is recommended and prescribed with probability 1 in every state. We call this a Beta equilibrium (BE). Of these two types of equilibria - the $\mathrm{CE}$ and the $\mathrm{BE}$ - the former better matches the empirical evidence on the effects of DTCA. Both types of equilibria imply an increase in the prescribing of $\operatorname{drug} \beta$ relative to the PKBE (i.e., a market stealing effect), but only the CE predicts an increase in patient-doctor conflict, which is a well-known side-effect of DTCA (Kravitz et al., 2003). ${ }^{14}$

\subsection{Purifying equilibria}

At the cost of some elaboration of the model, it is possible to dispense with the need for patient randomisation in equilibrium. ${ }^{15}$ We will sketch the required elaboration here, though the rest of the paper works with the nonelaborated model for simplicity.

Suppose that patients are heterogeneous in their conflict costs, with $c_{p}$ distributed according to a continuous distribution function $G$, which is strictly increasing on its support. If the doctor cannot observe $c_{p}$ (but knows $G$ ) patient responses may be stochastic from the doctor's perspective even if no patient type actually randomises.

Let $H$ denote the distribution function for $\bar{s}$ induced by the distribution $G$ over conflict costs. ${ }^{16}$ Consider a putative equilibrium in which there is some

\footnotetext{
${ }^{14}$ The Supplementary Material discusses the robustness of our conclusions to allowing BE-inducing DTCA.

${ }^{15}$ Our thanks to an anonymous referee for suggesting this possibility.

${ }^{16}$ Note that $\bar{s}>0$ when $c_{p}=0$ by Assumption 1 . Moreover, $\bar{s}$ is strictly increasing in
} 
$s^{*} \in(0, \tau]$ such that the doctor chooses $r=\hat{\alpha}$ when $s \in\left[\omega, s^{*}\right)$ and $r=\hat{\beta}$ when $s \in\left(s^{*}, 1\right]$. All patient types will accept the latter recommendation ( since $s^{*}>0$ ). In the putative equilibrium, the doctor will expect the former recommendation to be accepted with probability $1-H\left(s^{*}\right)$ (Lemma 2 ). The necessary and sufficient condition for this putative equilibrium to exist is that

$$
s^{*}-\tau=H\left(s^{*}\right)\left(s^{*}-\tau-c_{d}\right)
$$

so that the doctor is indifferent about which recommendation to make in state $s^{*}$ (Lemma 1). This indifference condition may be written

$$
H\left(s^{*}\right)=\frac{\left(\tau-s^{*}\right)}{\left(\tau-s^{*}\right)+c_{d}}
$$

Since $c_{d}>0$, it is easy to see that equation (6) has a unique solution in $(0, \tau]$ : the left-hand side increases (weakly) from $H(0)=0$; the right-hand side is strictly decreasing from a strictly positive value at $s^{*}=0$ to a value of zero at $s^{*}=\tau$. See Figure 1 .

The solution to (6) generates a set of equilibria with the following common features: the doctor recommends drug $\alpha$ when $s \in\left[\omega, s^{*}\right)$ and drug $\beta$ when $s \in\left(s^{*}, 1\right]$; all patient types accept recommendation $\hat{\beta}$; all patients with $c_{p}$ such that $\bar{s}$ in (1) exceeds (respectively, falls short of) $s^{*}$ accept (respectively, reject) recommendation $\hat{\alpha}$. The probability of rejecting $r=\hat{\alpha}$ is thus given by $(6)$.

With this "purified" equilibrium, persuasive DTCA will shift the distribution $H$, which will change the solution to (6). For example, if the common patient beliefs described by $F$ become more optimistic about drug quality, then $\bar{s}$ will fall for every patient type and the new $H$ will lie to the left (firstorder stochastically dominate) the old. From Figure 1 we observe that this will give a lower solution for $s^{*}$, so there will be more states in which the doctor recommends $\operatorname{drug} \beta$ and more patients who reject $r=\hat{\alpha}$. In other words, persuasive DTCA raises the profit of the drug firm through a market stealing effect.

$c_{p}$ when $\bar{s}<1$. It follows that the lower bound on the support of $H$ is strictly positive, and that $H$ is continuous at any $\bar{s}<1$. 


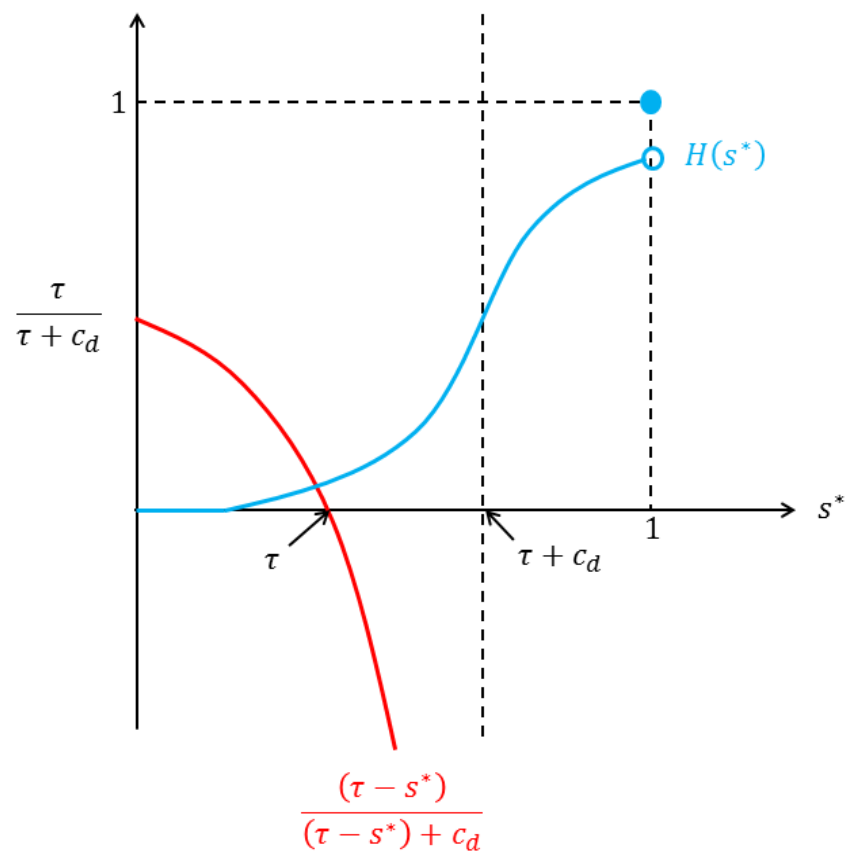

Figure 1: Solving (6)

\section{DTCA as a signal of quality}

Suppose that drug $\beta$ comes in $n$ possible quality levels, indexed by $q \in$ $\{1, \ldots, n\}$, and a drug of quality $q$ has efficacy distribution $F^{q}$. The quality of $\operatorname{drug} \beta$ is ascertained through scientific evidence (i.e., RCT data). This trial data is known to the pharmaceutical company and to the physician, ${ }^{17}$ but not to the patient.

In the consultation game, the distribution $F$ represents the patient's prior beliefs about drug efficacy. These beliefs will incorporate any information the patient has gleaned from DTCA. In the absence of DTCA, we assume that patients assign prior probability $\pi_{q} \in(0,1)$ to quality $q$. Therefore, if DTCA

${ }^{17}$ Azoulay (2002) finds that prescribing does respond to scientific evidence on the efficacy of a drug. 
is banned, the patient's beliefs are represented by

$$
F=\sum_{q=1}^{n} \pi_{q} F^{q}
$$

Assumption 2 For each $q \in\{1, \ldots, n\}$, the distribution $F^{q}$ is continuous with support $\left[\omega_{q}, 1\right]$ for some $\omega_{q} \in[\omega, 0)$. It is strictly increasing on its support and satisfies $F\left(\omega_{q}\right)=0$.

We index drugs such that lower $q$ values imply higher quality - think of $q$ as the drug's quality rank. We assume that drug $q$ has a quality advantage over drug $q+1$ in the following sense: ${ }^{18}$

Assumption 3 Distribution supports satisfy

$$
\underline{s}=\omega_{n}<\omega_{n-1}<\cdots<\omega_{1}
$$

and, for every $q \in\{1,2, \ldots, n-1\}$, distribution $F^{q}$ strictly dominates distribution $F^{q+1}$ in the sense of the reverse hazard rate order. That is,

$$
\frac{F^{q}(s)}{F^{q+1}(s)}
$$

is strictly increasing on $\left(\omega_{q}, 1\right)$. In particular (see Shaked and Shanthikumar, 1994, Section 1.B.6):

$$
\frac{1}{F^{q}(z)} \int_{\underline{s}}^{z} s d F^{q}(s)>\frac{1}{F^{q+1}(z)} \int_{\underline{s}}^{z} s d F^{q+1}(s)
$$

for any $z \in\left(\omega_{q}, 1\right)$.

${ }^{18}$ Assumption 3 implies that $F^{q}$ first-order stochastically dominates (FOSD) $F^{q+1}$, but is stronger. We thank a referee for pointing out the need for this stronger condition. Note that since $F^{q}(1)=F^{q+1}(1)$, we can only have $F^{q}(s) / F^{q+1}(s)$ strictly increasing on $\left(\omega_{q}, 1\right)$ if $\omega_{q+1}<\omega_{q}$. Hence the restriction on the supports. 
Condition (7) says that, conditional on learning $s \in[\underline{s}, z]$, patients expect a higher net health benefit from drug $\beta$ when it is of quality (rank) $q$ than when it is of quality (rank) $q+1$.

Defining

$$
\bar{s}_{q}=\sup \left\{s^{\prime} \in S \mid \int_{\underline{s}}^{s^{\prime}}\left(s-c_{p}\right) d F^{q}(s) \leq 0\right\},
$$

an immediate consequence of Assumption 3 is that $\bar{s}_{q} \leq \bar{s}_{q+1}$ for any $q \in$ $\{1,2, \ldots, n-1\}$, with strict inequality if $\bar{s}_{q}<1$.

Suppose, for example, that $\bar{s}_{1}<\tau \leq \bar{s}$, where $\bar{s}$ is defined by (1) for $F=\sum_{q=1}^{n} \pi_{q} F^{q}$. If DTCA is banned, the consultation game possesses a PKBE (since $\tau \leq \bar{s}$ ) and the physician writes her favoured prescription in every state. However, if $\beta$ 's true quality is $q=1$ and the producer of drug $\beta$ ("firm $\beta$ ") could credibly convey this information to the patient, the PKBE would no longer be viable (since $\bar{s}_{1}<\tau$ ) and the CE may be played instead. This would increase revenue for firm $\beta$.

Suppose that firm $\beta$ has an opportunity to advertise to patients prior to patients consulting their doctors. The sequence of moves is now as follows:

1. Nature chooses the drug quality. Quality $q$ is chosen with probability $\pi_{q}$.

2. Firm $\beta$ observes $q$ and choses an action from $\mathcal{A}=\{\varnothing, 1,2, \ldots, n\}$, where $\varnothing$ denotes no advertising and $a \in\{1,2, \ldots, n\}$ is an advertised claim that $q=a^{19}$

3. Nature chooses the state $s \in S$ according to distribution $F^{q}$ (where $q$ is the quality chosen at stage 1).

4. The physician observes $q, s$ and $a$ and makes her recommendation to the patient $(r \in\{\hat{\alpha}, \hat{\beta}\})$.

\footnotetext{
${ }^{19} \mathrm{We}$ assume that the producers of $\alpha$ do not advertise. If drug $\alpha$ is a well-known, offpatent medication and competitively supplied, then it is reasonable to suppose that the producers of $\alpha$ have no incentive to advertise.
} 
5. The patient observes $a$ and $r$ and decides on treatment $(t \in\{\alpha, \beta\})$.

The payoffs to the physician and patient are the same as in the consultation game. Firm $\beta$ 's payoff depends on its sales and its advertising costs. We shall treat stages 3-5 of the game as a "representative" consultation, and assume that firm $\beta$ earns total revenue (net of production costs) equal to $R$ if $\beta$ is prescribed at the end of the typical consultation. If action $a \in \mathcal{A} \backslash\{\varnothing\}$ is chosen in stage 2 , the drug firm pays an additional advertising cost $K>0$. Advertisements are audited with probability $\chi \in[0,1]$ and if $a \neq q$, a fine $\phi \geq 0$ is imposed.

The drug firm is risk neutral, maximising expected profit. If drug $\beta$ is of quality $q$ and prescribed with probability $\mu$ in the typical consultation, its expected profit is

$$
\mu R-\mathbb{I}[a \neq \varnothing] K-\mathbb{I}[a \notin\{\varnothing, q\}] \chi \phi .
$$

Consider a scenario in which the PKBE exists (and is played) when DTCA is banned. Furthermore, suppose that $\bar{s}_{1}<\tau$. Then DTCA may permit a type $q=1$ firm to credibly signal its quality to patients - and increase sales of its drug - as follows. Suppose that patients expect the drug firm to advertise if and only if $q=1$. If no DTCA is observed, they therefore infer that $q>1$ and follow the PKBE strategy in the consultation phase. ${ }^{20}$ If they observe $a=1$, they infer that $q=1$. Since $\bar{s}_{1}<\tau$ the PKBE cannot exist, but the CE (for $\left.F=F^{1}\right)$ does.

Are patient beliefs rational in this scenario? Let

$$
\sigma_{q}^{*}=\frac{\left(\tau-\bar{s}_{q}\right)}{\left(\tau-\bar{s}_{q}\right)+c_{d}}
$$

denote the probability that $\hat{\alpha}$ is rejected in a $\mathrm{CE}$ of the consultation game with $F=F^{q}$. It is optimal for a type $q=1$ firm to choose $a=1$ iff

$$
\left[F^{1}(\tau)-\left(1-\sigma_{1}^{*}\right) F^{1}\left(\bar{s}_{1}\right)\right] R \geq K
$$

\footnotetext{
${ }^{20}$ Recall that the PKBE exists when patients know only that $q \geq 1$ (DTCA is banned). If DTCA allows them to exclude $q=1$, they will be no less willing to follow the advice of a doctor who is playing the PKBE strategy (and to accept recommendation $\hat{\alpha}$ in particular).
} 
The left-hand side is the difference between expected revenue under the CE, which is

$$
\left[1-F^{1}\left(\bar{s}_{1}\right)+\sigma_{1}^{*} F^{1}\left(\bar{s}_{1}\right)\right] R
$$

and expected revenue under the PKBE, which is

$$
\left[1-F^{1}(\tau)\right] R
$$

It is optimal for a type $q=1$ firm to choose $a=1$ iff this difference is at least as high as its advertising cost, $K$. Similarly, it is optimal for a type $q>1$ firm not to choose $a=1 \mathrm{iff}$

$$
\left[F^{q}(\tau)-\left(1-\sigma_{1}^{*}\right) F^{q}\left(\bar{s}_{1}\right)\right] R \leq K+\chi \phi
$$

Patient beliefs are therefore rational provided $K$ is not too high - so (8) is satisfied - and $\chi \phi$ is high enough to ensure (9). Under these conditions, DTCA is credible. It is also effective - it switches behaviour in the consultation from the PKBE to the CE strategies for $F=F^{1}$, thereby increasing sales of $\operatorname{drug} \beta$.

The following Proposition and its Corollary formalise this intuition.

Proposition 3 Suppose $\bar{s}_{1}<\tau \leq \bar{s}$, where $\bar{s}$ is defined by (1) for $F=$ $\sum_{q=1}^{n} \pi_{q} F^{q}$, and further suppose that

$$
K \leq\left[F^{1}(\tau)-\left(1-\sigma_{1}^{*}\right) F^{1}\left(\bar{s}_{1}\right)\right] R
$$

Letting

$$
\underline{x}=\max _{q \in\{2, \ldots, n\}}\left[F^{q}(\tau)-\left(1-\sigma_{1}^{*}\right) F^{q}\left(\bar{s}_{1}\right)\right] R-K
$$

the following is an equilibrium strategy profile iff $\chi \phi \geq \underline{x}$ :

The Drug Firm: advertises truthfully if $q=1$ and does not advertise otherwise.

The Physician: follows her PKBE strategy if $a \in\{\varnothing, 2, \ldots, n\}$, recommends $\beta$ if $a=1$ and $s>\bar{s}_{1}$, and recommends $\alpha$ otherwise.

The Patient: always accepts recommendation $\hat{\beta}$, accepts recommendation $\hat{\alpha}$ if $a \in\{\varnothing, 2, \ldots, n\}$, and accepts recommendation $\hat{\alpha}$ with probability $1-\sigma_{1}^{*}$ if $a=1$. 


\section{Corollary 1 If}

$$
K<\max _{q \in\{2, \ldots, n\}}\left[F^{q}(\tau)-\left(1-\sigma_{1}^{*}\right) F^{q}\left(\bar{s}_{1}\right)\right] R
$$

the equilibrium in Proposition 3 requires $\chi \phi>0$.

The condition $\bar{s}_{1}<\tau \leq \bar{s}$ in Proposition 3 ensures that the consultation game possesses a PKBE when patient beliefs are given by $F=\sum_{q=1}^{n} \pi_{q} F^{q}$, while if patient beliefs are $F=F^{1}$ then the CE exists but not the PKBE. Condition (10) ensures that the highest quality drug firm is prepared to advertise if doing so switches play from the PKBE strategies to the CE strategies (for $F=F^{1}$ ) in the consultation phase.

Proposition 3 makes it clear that it is not only truth-in-advertising regulation that supports the credibility of DTCA. The direct cost of advertising also plays a role, as in Milgrom and Roberts (1986). If condition (11) in Corollary 1 fails, then DTCA may still occur in equilibrium even if content is unregulated. However, excluding this special case, truth-in-advertising regulation is critical to the credibility of DTCA (i.e., $\underline{x}>0$ ).

Closer inspection of the equilibrium in Proposition 3 also reveals an important role for the physician in sustaining DTCA. If $a=1$ and $s<\bar{s}_{1}$ the doctor will try to dissuade the patient from taking drug $\beta$ and will be successful with probability $1-\sigma_{1}^{*}$. This discourages lower quality drug producers from falsely claiming that $q=1$. Assumption 3 implies that $F^{q}$ FOSD $F^{q+1}$ for any $q<n$, so the doctor's reluctance to prescribe $\beta$ when $s<\bar{s}_{1}$ is more costly for a low-quality drug producer than for a higher quality producer. This also contributes to the credibility of DTCA.

We do not attempt a complete welfare analysis, as our model omits too many of the market failures that complicate the picture (such as the moral hazard effects of insurance on consumer demand for pharmaceuticals). However, it is interesting to compare the payoffs of the players in the equilibrium of Proposition 3 relative to their payoffs in the default scenario in which DTCA is banned (or when $\chi \phi$ is too low to support it). In the latter scenario, doctors and patients get their PKBE payoffs, and the drug manufacturer gets the expected profit from the PKBE level of prescribing. 
If the drug firm is of type $q>1$ then no player is affected by moving from the default scenario to the equilibrium in Proposition 3. However, if $q=1$ then the physician is made worse off - she no longer gets her way in the consultation - while the drug firm is (weakly) better off - it (weakly) increases its expected profit through the use of DTCA. The patient's expected welfare (under the true distribution $F^{1}$ ) changes by:

$$
\begin{aligned}
& \int_{\underline{s}}^{\tau} s d F^{1}(s)-\left(1-\sigma_{1}^{*}\right) \int_{\underline{s}}^{\bar{s}_{1}} s d F^{1}(s)-\sigma_{1}^{*} F^{1}\left(\bar{s}_{1}\right) c_{p} \\
> & \int_{\underline{s}}^{\bar{s}_{1}} s d F^{1}(s)-\left(1-\sigma_{1}^{*}\right) \int_{\underline{s}}^{\bar{s}_{1}} s d F^{1}(s)-\sigma_{1}^{*} F^{1}\left(\bar{s}_{1}\right) c_{p} \\
= & \sigma_{1}^{*} F^{1}\left(\bar{s}_{1}\right) c_{p}-\sigma_{1}^{*} F^{1}\left(\bar{s}_{1}\right) c_{p}=0
\end{aligned}
$$

where the inequality uses the fact that $0<\bar{s}_{1}<\tau$ and the first equality uses the definition of $\bar{s}_{1}$. Therefore, patients and drug manufacturers gain at the expense of physicians (and their employers).

\subsection{False DTCA}

In this section we show that false advertising can also be sustained in equilibrium. Of course, the notion of equilibrium precludes patients being mislead. They are aware that some DTCA may be false and form their beliefs accordingly. Nevertheless, it may be profitable for some types to advertise falsely in equilibrium.

To describe such an equilibrium, it is useful to define

$\bar{s}_{12}=\sup \left\{s^{\prime} \in S \mid \pi_{1} \int_{\underline{s}}^{s^{\prime}}\left(s-c_{p}\right) d F^{1}(s)+\pi_{2} \int_{\underline{s}}^{s^{\prime}}\left(s-c_{p}\right) d F^{2}(s) \leq 0\right\}$

and

$$
\sigma_{12}^{*}=\frac{\left(\tau-\bar{s}_{12}\right)}{\left(\tau-\bar{s}_{12}\right)+c_{d}} .
$$

If patients learn via DTCA that $q \in\{1,2\}$, then they form the belief

$$
F^{12}=\left(\frac{\pi_{1}}{\pi_{1}+\pi_{2}}\right) F^{1}+\left(\frac{\pi_{2}}{\pi_{1}+\pi_{2}}\right) F^{2} .
$$

For these beliefs, the consultation game has a CE provided $\tau>\bar{s}_{12}$ and recommendation $\hat{\alpha}$ is rejected with probability $\sigma_{12}^{*}$ in that $\mathrm{CE}$. 
Proposition 4 Suppose $\bar{s}_{12}<\tau \leq \bar{s}$ and

$$
K \leq\left[F^{1}(\tau)-\left(1-\sigma_{12}^{*}\right) F^{1}\left(\bar{s}_{12}\right)\right] R
$$

Letting

$$
\underline{x}_{12}=\max _{q \in\{3, \ldots, n\}}\left[F^{q}(\tau)-\left(1-\sigma_{12}^{*}\right) F^{q}\left(\bar{s}_{12}\right)\right]-K
$$

and

$$
\bar{x}_{12}=\left[F^{2}(\tau)-\left(1-\sigma_{12}^{*}\right) F^{2}\left(\bar{s}_{12}\right)\right]-K
$$

the following is an equilibrium strategy profile iff $\chi \phi \in\left[\underline{x}_{12}, \bar{x}_{12}\right]$ :

The Drug Firm: posts advertisement $a=1$ if $q \in\{1,2\}$ and does not advertise otherwise.

The Physician: follows her PKBE strategy if $a \in\{\varnothing, 2,3, \ldots, n\}$, recommends $\beta$ if $a=1$ and $s>\bar{s}_{12}$, and recommends $\alpha$ otherwise.

The Patient: always accepts recommendation $\hat{\beta}$, accepts recommendation $\hat{\alpha}$ if $a \in\{\varnothing, 2,3, \ldots, n\}$, and accepts recommendation $\hat{\alpha}$ with probability $1-\sigma_{12}^{*}$ if $a=1$.

\section{Corollary 2 If}

$$
K<\max _{q \in\{3, \ldots, n\}}\left[F^{q}(\tau)-\left(1-\sigma_{12}^{*}\right) F^{q}\left(\bar{s}_{12}\right)\right] R
$$

the equilibrium in Proposition 4 requires $\chi \phi>0$

In addition to exhibiting the possibility of false advertising in equilibrium, Proposition 4 also illustrates the fact that the relationship between regulation and the level of DTCA is not straightforward. If $\chi \phi<\underline{x}_{12}$ this equilibrium does not exist, but strengthening regulation such that $\chi \phi \in\left[\underline{x}_{12}, \bar{x}_{12}\right]$ would create the potential for false DTCA to arise via such an equilibrium. Regulation has a direct deterrent effect but it may also have an indirect credibilityenhancing effect. Intermediation by the doctor cannot completely undo the latter, given that her preferences are not perfectly aligned with the patient's.

Once again, the credibility of advertising rests on both the direct advertising cost $K$ and the expected fine $\chi \phi$. Provided $K$ is not too high, the role of regulation is critical (Corollary 2). 
As with the equilibrium of Proposition 3, the doctor also plays an important role in supporting the credibility of DTCA. In fact, her role is even more important here. If $\sigma_{12}^{*} \rightarrow 1$ (e.g., if $c_{d} \rightarrow 0$ ), the doctor's attempts to dispute DTCA are ignored by the patient and the equilibrium of Proposition 4 cannot exist. To see this, note that when $\sigma_{12}^{*}=1$

$$
\underline{x}_{12}=\max _{q \in\{3, \ldots, n\}}\left[F^{q}(\tau)\right]-K
$$

and

$$
\bar{x}_{12}=\left[F^{2}(\tau)\right]-K
$$

Since $F^{2}(\tau)<F^{q}(\tau)$ for any $q>2$ (an implication of Assumption 3), we have $\bar{x}_{12}<\underline{x}_{12}$. Intuitively, the doctor's ability to reduce prescribing of drug $\beta$ when $s<\bar{s}_{12}$ is what allows drug quality to affect incentives for false advertising: lower quality drugs suffer more from the doctor's push-back, as they face a higher probability that $s<\bar{s}_{12}$. If the doctor cannot effectively dispute DTCA, then all types $q>1$ have the same incentive to make the false claim $a=1$. This undermines the credibility of DTCA.

In general, the necessary and sufficient condition for $\underline{x}_{12} \leq \bar{x}_{12}$ is

$$
F^{q}(\tau)-\left(1-\sigma_{12}^{*}\right) F^{q}\left(\bar{s}_{12}\right) \leq F^{2}(\tau)-\left(1-\sigma_{12}^{*}\right) F^{2}\left(\bar{s}_{12}\right)
$$

for all $q>2$. This says that a type 2 firm experiences a weakly higher increase in expected revenue than a type $q>2$ firm, when moving from the PKBE to the $\mathrm{CE}$ (for patient beliefs $F^{12}$ ). This means that a type 2 firm has more incentive to send misleading DTCA than any lower quality type. The Supplementary Material contains a simple example for which $\underline{x}_{12}<\bar{x}_{12}$.

We may re-express (14) in the equivalent form:

$$
\left[\frac{F^{q}(\tau)}{F^{2}(\tau)}-1\right] \leq\left(1-\sigma_{12}^{*}\right) \frac{F^{2}\left(\bar{s}_{12}\right)}{F^{2}(\tau)}\left[\frac{F^{q}\left(\bar{s}_{12}\right)}{F^{2}\left(\bar{s}_{12}\right)}-1\right]
$$

Recall that Assumption 3 implies

$$
\frac{F^{q}(\tau)}{F^{2}(\tau)} \leq \frac{F^{q}\left(\bar{s}_{12}\right)}{F^{2}\left(\bar{s}_{12}\right)}
$$


Since

$$
\left(1-\sigma_{12}^{*}\right) \frac{F^{2}\left(\bar{s}_{12}\right)}{F^{2}(\tau)} \leq 1
$$

condition (15) requires that the difference

$$
\frac{F^{q}\left(\bar{s}_{12}\right)}{F^{2}\left(\bar{s}_{12}\right)}-\frac{F^{q}(\tau)}{F^{2}(\tau)}
$$

be sufficiently large, with the required difference increasing in $\sigma_{12}^{*}$. The less effective is the push-back from the doctor (i.e., the higher is $\sigma_{12}^{*}$ ), the harder it is to sustain the equilibrium in Proposition 4.

Let us compare the player payoffs in the equilibrium of Proposition 4 to those in a scenario in which DTCA is banned (or a scenario in which $\chi \phi$ is too low, or too high, to support it). If $q>2$, there is no effect. If $q \in\{1,2\}$ the payoff to the drug firm increases and that to the doctor falls. For any given $q \in\{1,2\}$, the patient's expected welfare (under the true distribution $F^{q}$ ) changes by:

$$
\int_{\underline{s}}^{\tau} s d F^{q}(s)-\left(1-\sigma_{12}^{*}\right) \int_{\underline{s}}^{\bar{s}_{12}} s d F^{q}(s)-\sigma_{12}^{*} F^{q}\left(\bar{s}_{12}\right) c_{p}
$$

relative to the scenario with no DTCA. This expression is strictly positive if $q=1$ (i.e., if DTCA conveys truthful information) since

$$
\begin{aligned}
& \int_{\underline{s}}^{\tau} s d F^{1}(s)-\left(1-\sigma_{12}^{*}\right) \int_{\underline{s}}^{\bar{s}_{12}} s d F^{1}(s)-\sigma_{12}^{*} F^{1}\left(\bar{s}_{12}\right) c_{p} \\
> & \sigma_{12}^{*} \int_{\underline{s}}^{\bar{s}_{12}} s d F^{1}(s)-\sigma_{12}^{*} F^{1}\left(\bar{s}_{12}\right) c_{p} \\
\geq & \sigma_{12}^{*} F^{1}\left(\bar{s}_{12}\right) c_{p}-\sigma_{12}^{*} F^{1}\left(\bar{s}_{12}\right) c_{p}=0,
\end{aligned}
$$

where we have used the fact that $\bar{s}_{1} \leq \bar{s}_{12}<\tau$. However, if $q=2$ it is possible that (16) is negative. ${ }^{21}$ Nevertheless, the patient's expected welfare

${ }^{21}$ Think of a situation in which $\bar{s}_{1}$ is very close to $\tau, q=2$ corresponds to a very low quality drug (so $\bar{s}_{2}$ is very high) but $\pi_{2} /\left(\pi_{1}+\pi_{2}\right)$ is very small. 
change, conditional on the event $q \in\{1,2\}$, is equal to:

$$
\begin{aligned}
& \int_{\underline{s}}^{\tau} s d F^{12}(s)-\left(1-\sigma_{12}^{*}\right) \int_{\underline{s}}^{\bar{s}_{12}} s d F^{12}(s)-\sigma_{12}^{*} F^{12}\left(\bar{s}_{12}\right) c_{p} \\
> & \sigma_{12}^{*} \int_{\underline{s}}^{\bar{s}_{12}} s d F^{12}(s)-\sigma_{12}^{*} F^{12}\left(\bar{s}_{12}\right) c_{p} \\
= & \sigma_{12}^{*} F^{12}\left(\bar{s}_{12}\right) c_{p}-\sigma_{12}^{*} F^{12}\left(\bar{s}_{12}\right) c_{p}=0 .
\end{aligned}
$$

Once again, patients and drug firms benefit from DTCA at the expense of doctors.

It is also straightforward to observe that $\bar{x}_{12} \leq \underline{x}$, since

$$
\left(1-\sigma_{12}^{*}\right) F^{2}\left(\bar{s}_{12}\right) \geq\left(1-\sigma_{1}^{*}\right) F^{2}\left(\bar{s}_{1}\right)
$$

It follows that if the equilibria of Propositions 3 and 4 both exist, then $\underline{x}_{12} \leq \bar{x}_{12} \leq \underline{x}$. In other words, higher expected fines are necessary to sustain the equilibrium with purely truthful DTCA than to sustain the equilibrium with false DTCA.

\section{Discussion}

Let us summarise our main conclusions. We consider a managed care environment in which doctors are imperfect agents of their patients. Assuming that patients always follow their physicians' recommendations when DTCA is banned (i.e., a PKBE is played), the introduction of DTCA may facilitate market stealing. Truth-in-advertising regulation plays an important role in supporting the credibility on which advertising relies to be effective.

Of course, we have only shown the possibility, not the certainty, of a market-stealing effect of DTCA. We have not characterised all equilibria of the model with DTCA. However, as we demonstrate in the Supplementary Material (Proposition 5), our conclusions are robust in the following sense: (i) whenever DTCA is observed in equilibrium, ${ }^{22}$ it always results in a strictly higher level of prescribing of drug $\beta$ than under a PKBE; and (ii) the expected

\footnotetext{
${ }^{22}$ Or rather, in a restricted equilibrium, as defined in the Supplementary Material.
} 
fine $\chi \phi$ must exceed a lower bound to support any equilibrium with purely truthful advertising, and must fall between an upper and lower bound for equilibria with both truthful and false advertising. ${ }^{23}$

Some key assumptions underpin our results. The remainder of this section discusses these assumptions.

We assume that physicians are biased against the new drug and that patients are aware of this. As discussed in the Introduction, there is evidence that managed care schemes place effective supply-side constraints on physicians' prescribing of expensive new drugs, and that patients are aware of this pressure.

We have also implicitly assumed that physicians cannot credibly convey information about the state $s$ to their patients - they merely recommend one drug or the other. Is this reasonable? Perhaps a doctor could present test results to convince her patient that $\operatorname{drug} \beta$ is unsuitable for him? We would argue, however, that while the test results may be verifiable to the patient, their proper interpretation is not. Specialised expertise is required to ascertain the relability of the test and to understand the significance of the results.

In the model with DTCA, we assume that drug advertisements must specify a particular quality level. This reflects U.S. regulations, which require firms to specify side-effects of the drug (and, if known, what can be done to lower the chance of experiencing them). The FDA sends out a warning letter if it believes that information has been left out. ${ }^{24}$ Therefore, it seems reasonable to restrict messages to precise claims about quality. One may think, for example, of quality as a list of side-effects, with $q$ a proper subset of $q+1$. The advertisement $a=q$ is the claim that $q$ is a complete list of all side-effects, not a claim that the elements of $q$ are amongst the side effects. We assume that the latter claim, which might be described by the

\footnotetext{
${ }^{23}$ Conditions for these bounds to be non-trivial may, of course, be substantive - as per Corollaries 1 and 2 .

${ }^{24}$ For instance, in the case of Vitrase, it sent out a warning letter in February 2005 because its advertisements did not include crucial risk information.
} 
set $\{q, q+1, \ldots, n\}$, is excluded by the nature of the regulations. ${ }^{25}$

The model also assumes a common fine for false advertising, irrespective of the level of falsehood. Relaxing this assumption would, we believe, only strengthen our results. Consider, for example, the equilibrium in Proposition 4. This relies on type $q=2$ being willing to lie, but not types $q>2$. If the latter falsehoods, being more serious, were punished more heavily, this would make the equilibrium even easier to support.

On the other hand, relaxing our assumption of a fixed advertising cost $K$ could potentially weaken results. If firms can affect this cost by choosing different levels of advertising intensity then patients may be able to infer quality from advertising expenditure, as in Milgrom and Roberts (1986). DTCA might then be an effective signal of quality even in the absence of regulation, since high-quality types can separate themselves by advertising more intensively. However, in practice, patients would be hard put to estimate the amount of money spent on advertising. We believe that our model, in which patients need only observe the fact, rather than the level, of advertising is more plausible. It is also useful for illustrating how advertising can be credible even without exploiting the intensity dimension.

We further assume that patients can observe the expected fine $\chi \phi$, and this assumption might also be questioned. The audit probability is particularly difficult to observe and need not remain constant over time. Indeed, concerns have been raised about the declining capacity of the FDA to properly administer DTCA regulations. For example, DTCA increased from $\$ 985 \mathrm{~m}$ in 1995 to $\$ 4,237 \mathrm{~m}$ in 2005 , while the number of FDA staff responsible for monitoring DTCA remained static over the same period. Consequently, the number of violation warning letters showed a marked decline (Donohue, Cevasco and Rosenthal, 2007).

If patients have an unreasonably optimistic expectation about the FDA's regulatory oversight there is potential for even greater patient-physician con-

\footnotetext{
${ }^{25}$ Of course, it is also interesting to consider how regulations might optimally distinguish between incomplete and false information, and between levels of falsehood. Either distinction could potentially be considered within a modified version of our model.
} 
flict from DTCA than our model might suggest. For example, suppose patients believe that $\chi \phi$ is large enough to support the equilibrium in Proposition 3 and therefore that firms have no incentive to advertise falsely. If, in reality, $\chi \phi$ is much smaller than patients' expectations, and low enough to induce some low quality types to make false claims, then patients will naïvely believe these claims. If a patient observes a false claim from a low quality firm, it is highly likely that the physician will recommend $\alpha$ and be challenged by the patient, whereas a properly informed patient would have accepted the physician's recommendation in the same state. There will consequently be higher levels of conflict and higher levels of prescribing of drug $\beta$. This (albeit informal) argument suggests that imperfectly understood regulation can induce more false advertising than a publicly announced repeal of all regulation.

Finally, our model does not include an explicit role for detailing. To the extent that detailing only reduces the physician's bias - as opposed to making the doctor positively inclined towards the new drug - our model still supports a market-stealing effect of DTCA. However, if detailing can render doctors positively inclined to the new drug - can reverse the physician's bias - then DTCA is best explained as part of a market-expanding strategy, as in Brekke and Kuhn (2006).

\section{Conclusion}

The appropriate regulation of DTCA is an important policy question. The existence of DTCA suggests that pharmaceutical firms believe it is effective in driving sales, and many detractors claim that it "distorts" prescribing towards more expensive drugs. In thinking about whether and how DTCA might influence prescribing, one must consider how drug advertising can be sufficiently credible to undermine the role of the "learned intermediary" the physician.

This paper is the first to present a signalling theory in which the credibility of DTCA is endogenised. The model also helps to explain the role of 
regulation in supporting the credibility of DTCA to a cynical patient population, and hence to sustaining the viability of advertising in equilibrium.

We do not undertake any formal welfare analysis here, so any conclusions about the appropriate regulatory responses to DTCA can only be speculative. Analysing the welfare effects of DTCA is not straightforward (Danzon and Keuffel, 2014). There are a plethora of market failures, including moral hazard in the insurance or HMO market, dynamic and static inefficiency in the market for new drugs, ${ }^{26}$ and asymmetric information in the market for phsyician services. A welfare analysis would need to consider all these market failures simultaneously.

Of course, if the policy concern is solely with false advertising, then one simple solution is to set the expected fine extremely high. But one of the lessons from our model is that marginal increments to expected fines might have unpredictable effects. The conditions for existence of equilibria with false DTCA impose a lower - as well as an upper - bound on the expected fine.

Some detractors of DTCA also worry about harm from truthful advertising. They argue that the physician performs a useful service by rationing expensive drugs so that scarce health dollars are used more efficiently in an environment with high levels of insurance coverage. If the policy aim is to reduce all DTCA, and an outright ban is infeasible, then complete de-regulation could be one option to consider as a potential means of undermining the credibility of advertising. ${ }^{27}$

\footnotetext{
${ }^{26} \mathrm{By}$ dynamic issues we mean the incentives for $\mathrm{R} \& \mathrm{D}$ while the static issues are about the monopolistic pricing of drugs resulting from patent protection.

${ }^{27}$ If such an approach were to be followed, it would be important to run a public awareness campaign to alert consumers to the unregulated status of DTCA - recall the discussion in Section 5.
} 


\section{References}

[1] Azoulay, P. (2002) "Do Pharmaceutical Sales Respond to Scientific Evidence?" Journal of Economics and Management Strategy 11(4), 551594.

[2] Berndt, E. L., T. Bui, D. Lucking-Reiley and G.L. Urban (1997) "The Roles of Marketing, Product Quality and Price Competition in the Growth and Composition of the US Anti-Ulcer Drug Industry" in The Economics of New Goods by T. F. Bresnahan and R. J. Gordon, Eds., University of Chicago Press: Chicago, 277-328.

[3] Berndt, E. L., T. Bui, D. H. Reiley and G.L. Urban (1995) "Information, Marketing and Pricing in the U.S. Anti-Ulcer Drug Market" American Economic Review 85(2), 100-105.

[4] Bradford, D., A. N. Kleit, P. J. Nietert et al. (2006) "How Direct-toConsumer Television Advertising for Osteoarthritis Drugs Affects Physicians' Prescribing Behavior" Health Affairs 25(5), 1371-1377.

[5] Brekke, K. R. and M. Kuhn (2006) "Direct to Consumer Advertising in Pharmaceutical Markets" Journal of Health Economics 25(1), 102-130.

[6] Calfee, J.E., C. Winston and R. Stempski (2002) "Direct-to-Consumer Advertising and the Demand for Cholesterol Reducing Drugs" Journal of Law and Economics XLV, 673-689.

[7] Cassels, A., M. Hughes, C. Cole et al. (2003) "Drugs in the News: An Analysis of Canadian Newspaper Coverage of New Prescription Drugs" CMAJ 168(9), 1133-1137.

[8] Danzon, P.M. and E.L. Keuffel (2014) "Regulation of the Pharmaceutical-Biotechnology Industry" in Economic Regulation and Its Reform: What Have We Learned? by Nancy L. Rose, Ed., University of Chicago Press: Chicago, 407-484. 
[9] Domino, M.E. and D.S. Salkever (2003) "Price Elasticity and Pharmaceutical Selection: The Influence of Managed Care" Health Economics 12(7), 565-586.

[10] Donohue, J.M., M. Cevasco and M.B. Rosenthal (2007) "A Decade of Direct-to-Consumer Advertising of Prescription Drugs" New England Journal of Medicine 357(7), 673-681.

[11] Epling, J. W., E. M. Mader, and C. P. Morley (2014) "Practice Characteristics and Prior Authorization Costs: Secondary Analysis of Data Collected by SALT-Net in 9 Central New York Primary Care Practices" BMC Health Services Research 14.1, 109

[12] Iizuka, T. (2004) "What Explains the Use of Direct-to-Consumer Advertising of Prescription Drugs?" Journal of Industrial Economics LII(3), 349-379.

[13] Iizuka, T. and G.Z. Jin (2005) "The Effect of Prescription Drug Advertising on Doctor Visits" Journal of Economics and Management Strategy 14(3), 701-727.

[14] Kao, A.C., D.C. Green, A.M. Zaslavsky et al. (1998) "The Relationship Between Method of Physician Payment and Patient Trust" Journal of the American Medical Association 280(19), 1708-1714.

[15] Kalyanaram, G. (2009) "The Endogenous Modeling of the Effect of Direct-to-Consumer Advertising of Prescription Drugs" International Journal of Pharmaceutical and Healthcare Marketing 3(2), 137-148.

[16] Kalyanaram, G. (2008) "The Order of Entry Effect in Prescription $(\mathrm{Rx})$ and Over-The-Counter (OTC) Pharmaceutical Drugs" International Journal of Pharmaceutical and Healthcare Marketing 2(1), 35-46.

[17] Kravitz R.L., R.A. Bell, R. Azari et al. (2003) "Direct Observation of Requests for Clinical Services in Office Practice: What Do Patients Want and Do They Get It?" Archives of Internal Medicine 163(14), 1673-1681. 
[18] Kravitz, R.L., R.M. Epstein, M.D. Feldman et al. (2005) "Influence of Patients' Requests for Direct-to-Consumer Advertised Antidepressants: A Randomized Controlled Trial" Journal of the American Medical Association 293(16), 1995-2002.

[19] Lanzi, T. and J. Mathis (2007) "Consulting an Expert with Potentially Conflicting Preferences" Theory and Decision 65, 185-204.

[20] Law, M. R., C.Y. Lu, S.B. Soumerai et al. (2010) "Impact of Two Medicaid Prior-Authorization Policies on Antihypertensive Use and Costs among Michigan and Indiana Residents Dually Enrolled in Medicaid and Medicare: Results of a Longitudinal, Population-Based Study" Clinical Therapeutics 32(4), 729-741.

[21] Ling, D.C., E.R. Berndt and M.K. Kyle (2002) "Deregulating Directto-Consumer Marketing of Prescription Drugs: Effects on Prescription and Over-The-Counter Product Sales" Journal of Law and Economics XLV, 691-723.

[22] Liu, Q. and S. Gupta (2011) "The Impact of Direct-to-Consumer Advertising of Prescription Drugs on Physician Visits and Drug Requests: Empirical Findings and Public Policy Implications" International Journal of Research in Marketing 28(3), 205-217.

[23] Milgrom, P. and J. Roberts (1986) "Prices and Advertising Signals of Product Quality" Journal of Political Economy 94(4), 796-821.

[24] Narayanan, S., R. Desiraju and P.K. Chintagunta (2004) "Return on Investment Implications for Pharmaceutical Promotional Expenditures: The Role of Marketing-Mix Interactions" Journal of Marketing 68, 90105.

[25] Nelson, P. (1974) "Advertising as Information" Journal of Political Economy 81, 729-754.

[26] Rosenthal, M.B., E.R. Berndt, J.M. Donohue et al. (2003) "Demand Effects of Recent Changes in Prescription Drug Promotion" in Frontiers 
in Health Policy Research, Volume 6 by D.M. Cutler and A.M. Garber, Eds., MIT Press: Cambridge, 1-26.

[27] Rubin, P.H. and J.L. Schrag (1999) "Mitigating Agency Problems by Advertising, With Special Reference to Managed Health Care" Southern Economic Journal 66(1), 39-60.

[28] Sauer, R.D. and K.B. Leffler (1990) "Did the Federal Trade Commission's Advertising Substantiation Program Promote More Credible Advertising?" American Economic Review 80(1), 191-203.

[29] Schlesinger, M. (2002) "A Loss of Faith: The Sources of Reduced Political Legitimacy for the American Medical Profession" The Milbank Quarterly 80(2), 185-235.

[30] Shaked, M. and J.G. Shanthikumar (1994) Stochastic Orders and Their Applications, Academic Press: London.

[31] Shuchman, M. (2007) "Drug Risk and Free Speech - Can Congress Ban Consumer Drug Ads?" New England Journal of Medicine 356, 22362239 .

[32] Spence, M., S. Teleki, C. Cheetham et al. (2005) "Direct to Consumer Advertising of Cox-2 Inhibitors: Effect on Appropriateness of Prescribing" Medical Care Research and Review 62, 544-559.

[33] U.S. General Accounting Office (2002) "Prescription Drugs: FDA Oversight of Direct-to-Consumer Advertising Has Limitations", Washington, DC.

[34] Vaithianathan, R., P. Hockey, T. Moore and D. Bates (2009) "Iatrogenic Effects of COX-2 Inhibitors in the US Population: Findings from the Medical Expenditure Panel Survey" Drug Safety 32(4), 335-343.

[35] Wosinska, M. (2002) "Just What the Patient Ordered? Direct-toConsumer Advertising and the Demand for Pharmaceutical Products" Harvard Business School Marketing Research Paper No. 02-04. 
[36] Wynia, M. K., J.B. Van Geest, D.S. Cummins and I.B. Wilson (2003) "Do Physicians Not Offer Useful Services Because of Coverage Restrictions?" Health Affairs 22(4), 190-197.

\section{Appendix}

Proof of Lemma 1. This follows from the fact that

$$
\mathbb{E}\left[u^{d}\left(r^{\prime}, t, s\right)-u^{d}(r, t, s) \mid s\right]=\left(\pi^{\prime}-\pi\right)(s-\tau)-\text { const. }
$$

is strictly increasing in $s$. (The "constant" is the difference in expected conflict costs for the two recommendations. This difference does not depend on s.) In other words, the difference in expected utility from recommending $r^{\prime}$ rather than $r$ in state $s$ is a strictly increasing function of $s$.

Proof of Lemma 2. We may re-write (2) in the following equivalent form:

$$
\int_{\underline{s}}^{s^{*}} s d F(s) \leq c_{p} F\left(s^{*}\right) \Leftrightarrow \int_{\underline{s}}^{s^{*}}\left(s-c_{p}\right) d F(s) \leq 0 .
$$

Hence, (2) implies $\bar{s} \geq s^{*}$. For the converse, it suffices to observe that if

$$
\int_{\underline{s}}^{s^{\prime}}\left(s-c_{p}\right) d F(s)>0
$$

then

$$
\int_{\underline{s}}^{s^{\prime \prime}}\left(s-c_{p}\right) d F(s)>0
$$

for any $s^{\prime \prime}>s^{\prime}$. Thus, if (2) did not hold, then $\bar{s}<s^{*}$ would necessarily follow, given that

$$
\int_{\underline{s}}^{s^{\prime}}\left(s-c_{p}\right) d F(s)
$$

is continuous in $s^{\prime}$.

Proof of Proposition 1. In any responsive and state monotonic equilibrium with $s^{*} \in(\omega, 1)$, we have $\sigma^{d}(s)=0$ when $s<s^{*}, \sigma^{d}(s)=1$ when $s>s^{*}$ 
and both recommendations occur with strictly positive probability - see the discussion following Lemma 1. It follows that $\sigma^{p}(\hat{\beta})$ is optimal for posterior beliefs that condition $F$ on $\left[s^{*}, 1\right]$, and $\sigma^{p}(\hat{\alpha})$ is optimal for posterior beliefs that condition $F$ on $\left[\underline{s}, s^{*}\right]$. It also follows that any PBE of this form will satisfy the IC, since there are no off-equilibrium recommendations.

Figure 2 depicts the optimal patient responses as a function of $s^{*} \in(\omega, 1)$. To construct Figure 2 we define

$$
\hat{s}=\inf \left\{s^{\prime} \in S \mid \int_{s^{\prime}}^{1}\left(s+c_{p}\right) d F(s) \geq 0\right\}
$$

This parameter determines the critical $s^{*}$ value at which the patient's optimal decision switches from accepting to rejecting $r=\hat{\beta}$, as verified in the following lemma. ${ }^{28}$

Lemma 3 Given $s^{*} \in(\omega, 1)$, the condition

$$
\frac{1}{\left[1-F\left(s^{*}\right)\right]} \int_{s^{*}}^{1} s d F(s) \geq-c_{p}
$$

is equivalent to $s^{*} \geq \hat{s}$.

Condition (18) is necessary and sufficient for the patient to accept $r=\hat{\beta}$ when posterior beliefs are given by $F$ conditioned on $\left[s^{*}, 1\right]$. Note that $\hat{s}<0$ by Assumption 1 .

From Figure 2 we see that responsiveness implies $s^{*} \in[\hat{s}, \bar{s}]$.

We may use

$$
\Delta=\sigma^{p}(\hat{\alpha})+\sigma^{p}(\hat{\beta})-1
$$

as a one-dimensional parameterisation of the optimal patient response to each $s^{*} \in(\omega, 1) \cap[\hat{s}, \bar{s}]$. To see why, note that if $s^{*} \in(\omega, 1) \cap[\hat{s}, \bar{s}]$ then the patient's optimal response takes one of the following mutually exclusive forms, depending on $s^{*}$ :

$$
\sigma^{p}(\hat{\alpha})=0 \text { and } \sigma^{p}(\hat{\beta})=1
$$

\footnotetext{
${ }^{28}$ Lemma 3 may be proved along similar lines to Lemma 2 - we leave the details to the reader.
} 

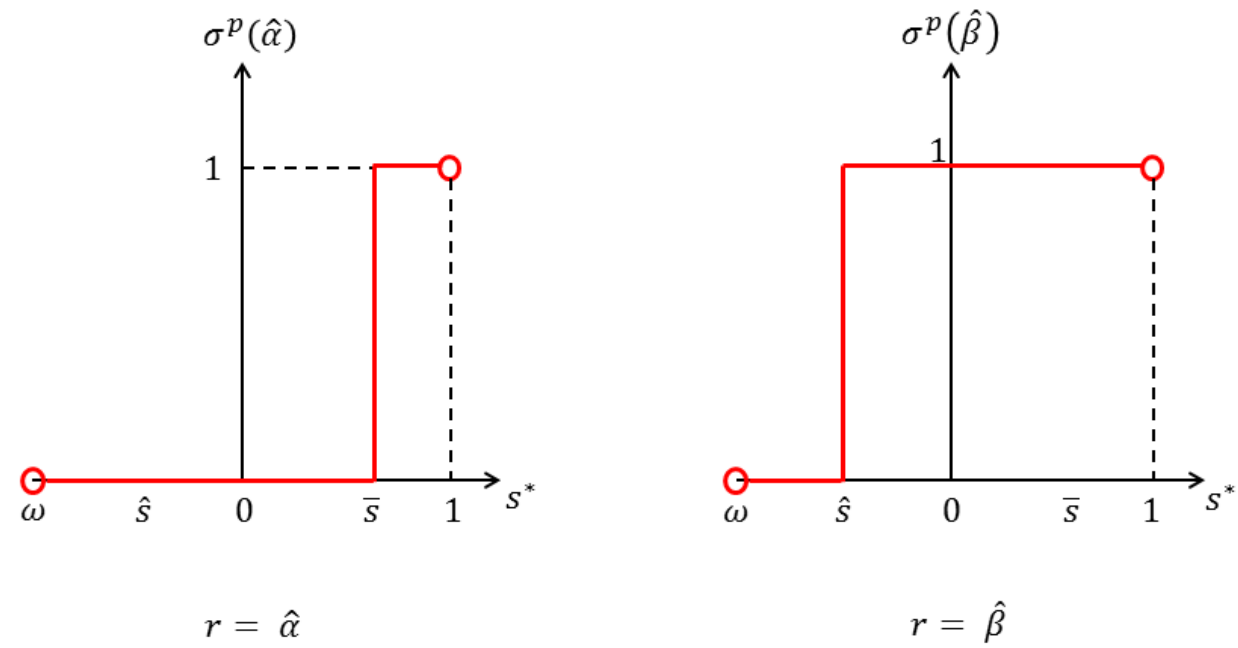

Figure 2: Patient best responses

$$
\begin{aligned}
& \sigma^{p}(\hat{\alpha}) \in(0,1] \text { and } \sigma^{p}(\hat{\beta})=1 \\
& \sigma^{p}(\hat{\alpha})=0 \text { and } \sigma^{p}(\hat{\beta}) \in[0,1)
\end{aligned}
$$

It is easily checked that $\Delta$ determines a one-to-one mapping from the set of scenarios described by (20)-(22) into $[-1,1] .{ }^{29}$ Scenario (20) gives $\Delta=0$; scenarios in $(21)$ generate $\Delta$ values in $(0,1]$; and scenarios in (22) generate $\Delta$ values in $[-1,0)$.

Figure 3 re-expresses the content of Figure 2 as a mapping from $s^{*} \in[\hat{s}, \bar{s}]$ to $\Delta \in[-1,1]$, assuming $[\hat{s}, \bar{s}] \subseteq(\omega, 1)$. It depicts the patient's optimal response given the physician strategy summarised by $s^{*}$.

It remains to determine the values of $s^{*}$ associated with the doctor's optimal responses to each $\Delta \in[-1,1]$. If the optimal $s^{*}$ lies in $(\omega, 1)$, then it coincides with the state at which the doctor is indifferent about which drug to recommend:

$$
\sigma^{p}(\hat{\alpha})\left(s^{*}-\tau-c_{d}\right)=\sigma^{p}(\hat{\beta})\left(s^{*}-\tau\right)-\left[1-\sigma^{p}(\hat{\beta})\right] c_{d}
$$

${ }^{29}$ It is onto if $(\omega, 1) \cap[\hat{s}, \bar{s}]=[\hat{s}, \bar{s}]$. 


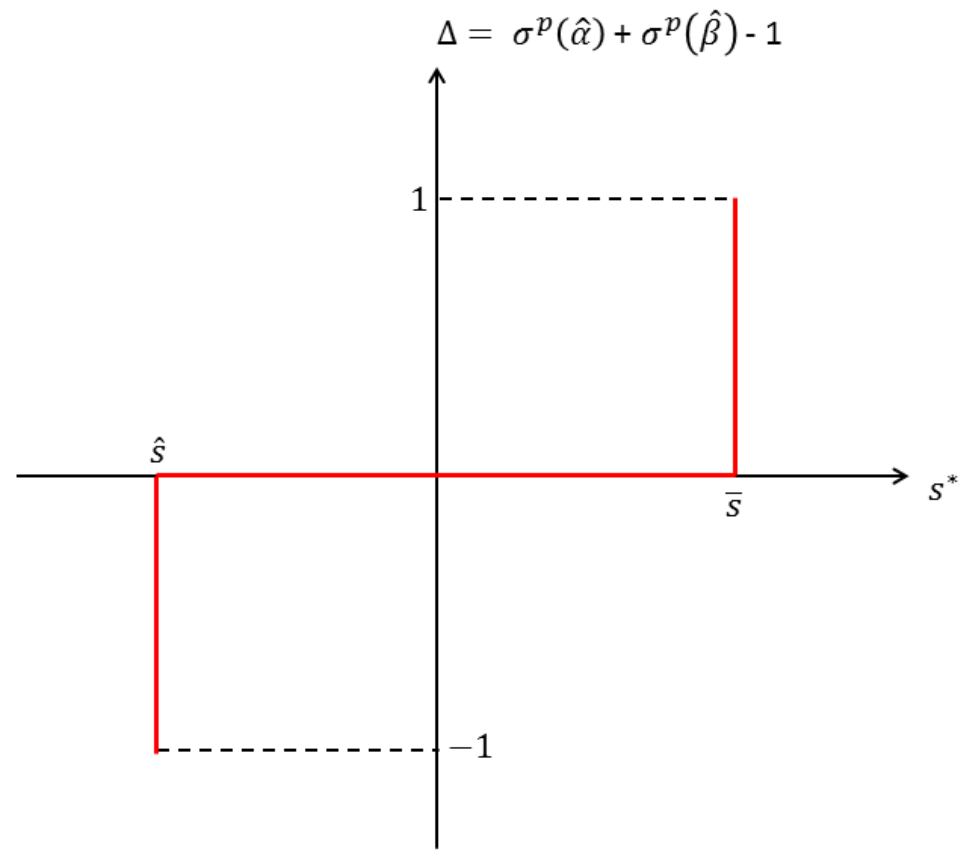

Figure 3: Patient best response (re-parameterised)

$$
\Leftrightarrow \quad s^{*}=\tau+\left[\frac{\Delta}{\sigma^{p}(\hat{\alpha})-\sigma^{p}(\hat{\beta})}\right] c_{d}
$$

Observe from (20) and (21) that

$$
\sigma^{p}(\hat{\alpha})-\sigma^{p}(\hat{\beta})=\Delta-1
$$

when $\Delta \in[0,1]$, and from (22) that

$$
\sigma^{p}(\hat{\alpha})-\sigma^{p}(\hat{\beta})=-\Delta-1
$$

when $\Delta \in[-1,0)$. Hence, for any scenario in (20)-(22), we have

$$
s^{*}=\tau+\left[\frac{\Delta}{|\Delta|-1}\right] c_{d}
$$

Figures 4 and 5 plot the physician response function (23) together with the patient response function from Figure 3, for the cases $\tau \leq \bar{s}$ and $\tau>\bar{s}$ respectively. 


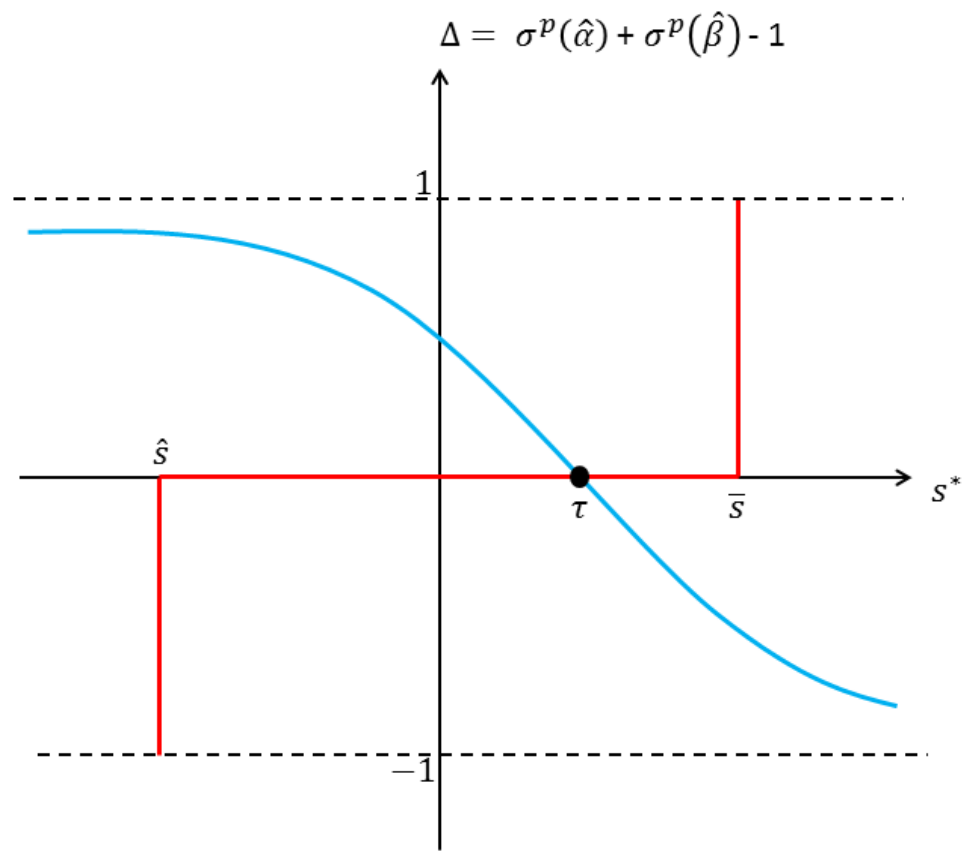

Figure 4: Equilibrium when $\tau \leq \bar{s}$

In each case, there is a unique equilibrium pair $\left(s^{*}, \Delta\right)$. When $\tau \leq \bar{s}$ this pair is $(\tau, 0)$. Since $\tau \in(\omega, 1)$, this pair induces a PKBE.

When $\tau>\bar{s}$ the equilibrium pair is

$$
\left(\bar{s}, \frac{(\tau-\bar{s})}{(\tau-\bar{s})+c_{d}}\right)
$$

Since $s^{*}=\bar{s} \in(0, \tau) \subseteq(\omega, 1)$, this pair induces a CE.

Proof of Proposition 2. Condition (5) implies $\tau \leq \bar{s}$, so a PKBE exists (Proposition 1).

It remains to show that the PKBE Pareto dominates any other equilibrium under condition (5). Rather than enumerate all equilibria, we proceed indirectly. We will divide (putative) equilibria into two classes - possibly empty - and show that, for each class, any member must be Pareto dominated by the PKBE.

We classify equilibria as follows: 


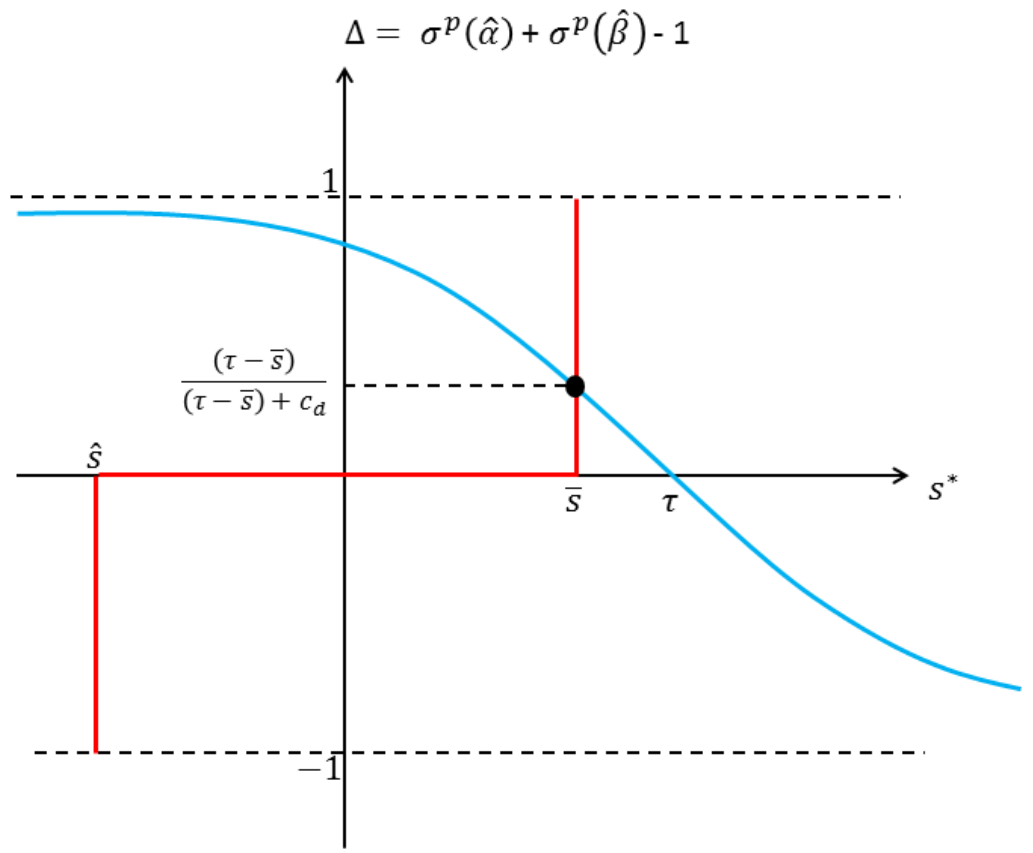

Figure 5: Equilibrium when $\tau>\bar{s}$

\section{Case I: Equilibria in which only one recommendation is made with} positive probability.

In such an equilibrium (if one exists), the sole equilibrium recommendation is made with probability 1 in every state. The patient's treatment lottery is therefore the same in every state. Since $\tau<1$, it follows that the physician is strictly worse off in any Case I equilibrium than in the $P K B E$. The physician obtains her favourite treatment in every state under the PKBE, and $\tau<1$ implies that this favourite treatment is $\alpha$ in some states and $\beta$ in others. An equilibrium in which the same treatment lottery occurs in every state must be strictly worse.

What about the patient? The patient's expected payoff under the $\mathrm{PKBE}$ is

$$
\int_{\tau}^{1} s d F(s)
$$

Denote this payoff by $\Pi$. Since $\tau<1$, we have $\Pi>0$ from Assumption 
1. Condition (5) implies

$$
\Pi \geq \int_{\underline{s}}^{1} s d F(s),
$$

So

$$
\Pi \geq \max \left\{0, \int_{\underline{s}}^{1} s d F(s)\right\}
$$

In a Case I equilibrium, the patient learns nothing from the doctor's equilibrium recommendation, so his expected equilibrium payoff is no higher than that from receiving his ex ante optimal treatment in all states without conflict. If he receives $\alpha$ in all states, his expected payoff is zero. If he receives $\beta$ in all states, his expected payoff is

$$
\int_{\underline{s}}^{1} s d F(s) .
$$

We therefore deduce from (24) that the patient's PKBE payoff is at least as high as his payoff in any Case I equilibrium.

It follows that the PKBE Pareto dominates any Case I equilibrium.

\section{Case II. Equilibria in which both recommendations are made with positive probability.}

We restrict attention to Case II equilibria which are responsive - otherwise the welfare analysis is the same as for Case I. By Lemma 1, there exists some $s^{*}$ with $F\left(s^{*}\right) \in(0,1)$ such that recommendation $r$ is made in states $s<s^{*}$, to which the patient responds by choosing $\beta$ with probability $\sigma^{p}(r)=\pi$, and recommendation $r^{\prime}$ is made in states $s>s^{*}$, to which the patient responds by choosing $\beta$ with probability $\sigma^{p}\left(r^{\prime}\right)=\pi^{\prime}>\pi$. Unless this equilibrium coincides with the PKBE (i.e., $s^{*}=\tau, r=\hat{\alpha}, r^{\prime}=\hat{\beta}, \pi=0$ and $\pi^{\prime}=1$ ), the physician is strictly worse off than under the PKBE. ${ }^{30}$ Once again, it suffices to show that the patient is no better off.

\footnotetext{
${ }^{30}$ More precisely, since $\tau \in(0,1)$, there must be a non-degenerate interval of states around $\tau$ in which the physician is strictly worse off. By Assumption 1, there is strictly positive ex ante probability of $s$ falling within this interval.
} 
Consider the patient's expected payoff in such an equilibrium (i.e., a responsive Case II equilibrium). We will show that this payoff is no greater than $\Pi$.

If $\pi^{\prime}<1$, it is optimal for the patient to choose $t=\alpha$ in response to either recommendation. It follows that her equilibrium expected payoff is no better than the payoff from receiving treatment $\alpha$ in every state without conflict. The latter payoff is zero, which is no greater than $\Pi$ - recall (24).

Suppose, then, that $\pi^{\prime}=1$. That is, the patient chooses $\beta$ in response to recommendation $r^{\prime}$.

If $r^{\prime}=\hat{\beta}$ (hence $r=\hat{\alpha}$ ), then either the equilibrium coincides with the PKBE or else $\pi>0$. (If $\pi=0$ the physician's optimal strategy implies $s^{*}=\tau$, so we have a PKBE.) If $\pi>0$, it is optimal for the patient to choose $t=\beta$ in response to either recommendation. It follows that his equilibrium payoff is no greater than if he were to receive treatment $\beta$ in every state without conflict. From Case I, we already know that $\Pi$ is at least as high as this - see (24) again.

Finally, consider equilibria with $r^{\prime}=\hat{\alpha}$ (hence $r=\hat{\beta}$ ).

If $\pi=0$ in such an equilibrium, then optimal physician behaviour implies $s^{*}=\tau$ - both recommendations provoke conflict, so the doctor recommends the one that results in her favoured treatment. In such an equilibrium (if it exists) the patient receives the same treatment as under the PKBE, but there is conflict with probability 1. The patient is clearly worse off in such an equilibrium than under the PKBE.

Finally, we show that an equilibrium with $r^{\prime}=\hat{\alpha}$ and $\pi \in(0,1)$ is precluded by condition (5). For such an equilibrium to exist, it is necessary that

$$
\int_{\underline{s}}^{s^{*}} s d F(s)=-F\left(s^{*}\right) c_{p}
$$

to ensure the patient is willing to randomise following recommendation 
$\hat{\beta}$. Since $\pi^{\prime}=1$, optimal physician behaviour implies that $s^{*}$ satisfies

$$
\begin{gathered}
s^{*}-\tau-c_{d}=\pi\left(s^{*}-\tau\right)-(1-\pi) c_{d} \\
\Leftrightarrow \quad s^{*}=\tau+\left(\frac{\pi}{1-\pi}\right) c_{d}
\end{gathered}
$$

In particular, $s^{*} \geq \tau$ so $(25)$ contradicts (5).

Thus, in any Case II equilibrium consistent with (5), the patient is no better off than under the PKBE.

Cases I and II exhaust all possibilities. In summary, we have shown that when condition (5) holds, any non-PKBE equilibrium is Pareto dominated by the PKBE. In particular, the doctor is strictly better off and the patient no worse off.

Proof of Proposition 3. The following Lemma will be useful in the sequel:

Lemma 4 If $\hat{F}=\sum_{q \in Q} \lambda_{q} F^{q}$ for some set $Q \subseteq\{1,2, \ldots, n\}$ and some constants $\lambda_{q} \in(0,1]$ satisfying $\sum_{q \in Q} \lambda_{q}=1$, then for any $t>0$

$$
\frac{1}{F^{\bar{q}}(t)} \int_{\underline{s}}^{t} s d F^{\bar{q}}(s) \leq \frac{1}{\hat{F}(t)} \int_{\underline{s}}^{t} s d \hat{F}(s) \leq \frac{1}{F^{q}(t)} \int_{\underline{s}}^{t} s d F^{q}(s)
$$

where $\underline{q}=\min Q$ and $\bar{q}=\max Q$.

Proof. We may write

$$
\frac{1}{\hat{F}(t)} \int_{\underline{s}}^{t} s d \hat{F}(s)=\sum_{q=1}^{n}\left(\frac{\lambda_{q} F^{q}(t)}{\hat{F}(t)}\right)\left[\frac{1}{F^{q}(t)} \int_{\underline{s}}^{t} s d F^{q}(s)\right]
$$

Since $\hat{F}(t)=\sum_{q=1}^{n} \lambda_{q} F^{q}(t)$, the right-hand side of (26) is a convex combination of the conditional means

$$
\frac{1}{F^{q}(t)} \int_{\underline{s}}^{t} s d F^{q}(s) .
$$

The result now follows by Assumption 3 . 
Consider the beliefs of the patient in the (putative) equilibrium, following the drug firm's move. If he observes $a=1$, he uses Bayes' Rule to form the belief $F^{1}$. The continuation strategies of the doctor and patient — which are those of the $\mathrm{CE}$ for $F=F^{1}$ - are therefore sequentially rational, since $\bar{s}_{1}<\tau$ (Proposition 1). If the patient observes $a=\varnothing$, he uses Bayes' Rule to form the belief

$$
F=\left(\frac{1}{1-\pi_{1}}\right) \sum_{q=2}^{n} \pi_{q} F^{q}
$$

This belief is more "pessimistic" than the prior, since only the highest quality drug firm has been excluded. More precisely, letting $\hat{F}=\sum_{q=1}^{n} \pi_{q} F^{q}$, Lemma 4 implies $^{31}$

$$
\frac{1}{F(\tau)} \int_{\underline{s}}^{\tau} s d F(s) \leq \frac{1}{\hat{F}(\tau)} \int_{\underline{s}}^{\tau} s d \hat{F}(s) .
$$

Since $\tau \leq \bar{s}$ (i.e., the consultation game possesses a PKBE based on the prior beliefs), we have

$$
\frac{1}{F(\tau)} \int_{\underline{s}}^{\tau} s d F(s) \leq c_{p}
$$

so the continuation strategies of the doctor and patient - which are those of the PKBE - are sequentially rational (Proposition 1).

If the patient observes $a \in\{2, \ldots, n\}$, he cannot use Bayes' Rule to form beliefs. We shall suppose that the patient forms the belief $F^{n}$ in these circumstances (but continues to believe that the physician will play according to the equilibrium strategies). Note that a drug firm of type $q=n$ could

${ }^{31}$ This follows from the observation that $\hat{F}=\pi_{1} F^{1}+\left(1-\pi_{1}\right) F$. We may therefore write

$$
\begin{gathered}
\frac{1}{\hat{F}(\tau)} \int_{\underline{s}}^{\tau} s d \hat{F}(s)= \\
\frac{\pi_{1} F^{1}(\tau)}{\hat{F}(\tau)}\left[\frac{1}{F^{1}(\tau)} \int_{\underline{s}}^{\tau} s d F^{1}(s)\right]+\frac{\left(1-\pi_{1}\right) F(\tau)}{\hat{F}(\tau)}\left[\frac{1}{F(\tau)} \int_{\underline{s}}^{\tau} s d F(s)\right]
\end{gathered}
$$

Moreover:

$$
\frac{1}{F(\tau)} \int_{\underline{s}}^{\tau} s d F(s) \leq \frac{1}{F^{2}(\tau)} \int_{\underline{s}}^{\tau} s d F^{2}(s)<\frac{1}{F^{1}(\tau)} \int_{\underline{s}}^{\tau} s d F^{1}(s)
$$

where the first inequality uses Lemma 4 and the second uses Assumption 3. The conditional mean of $F$ is therefore lower than that of $\hat{F}$. 
conceivably benefit from such a deviation, since $K<R$ by condition (10). It follows that these off-equilibrium beliefs satisfy the IC. Given these patient beliefs, the PKBE strategies are sequentially rational by Proposition 1, since we deduce $\tau \leq s_{n}$ from $\tau \leq \bar{s}$ and Lemma 4 .

It remains to check that the drug firm's strategy is optimal. The drug firm can either choose $a=1$ and induce the CE continuation (for patient beliefs $F=F^{1}$ ), or choose $a \in\{\varnothing, 2, \ldots, n\}$ and induce the PKBE continuation. Since advertising is costly, it is clear that the optimal choice must be $a=1$ or $a=\varnothing$. We must show that type $q=1$ (weakly) prefers $a=1$ while type $q>1$ (weakly) prefers $a=\varnothing$.

As per the discussion prior to Proposition 3, it is optimal for type $q=1$ to choose $a=1 \mathrm{iff}$

$$
\left[F^{1}(\tau)-\left(1-\sigma_{1}^{*}\right) F^{1}\left(\bar{s}_{1}\right)\right] R \geq K
$$

which is satisfied by assumption (10). It is optimal for type $q>1$ to choose $a=\varnothing$ iff

$$
\chi \phi \geq\left[F^{q}(\tau)-\left(1-\sigma_{1}^{*}\right) F^{q}\left(\bar{s}_{1}\right)\right] R-K
$$

Therefore, we have an equilibrium iff $\chi \phi \geq \underline{x}$.

Proof of Corollary 1. The right-hand side of (27) strictly exceeds zero for some $q>1$ iff (11).

Proof of Proposition 4. The proof follows similar lines to that of Proposition 3 .

A patient who observes $a=1$ forms belief

$$
F^{12}=\left(\frac{\pi_{1}}{\pi_{1}+\pi_{2}}\right) F^{1}+\left(\frac{\pi_{2}}{\pi_{1}+\pi_{2}}\right) F^{2}
$$

Since $\bar{s}_{12}<\tau$ it is sequentially rational for the doctor and patient to play the CE strategies (for belief $F^{12}$ ) in the consultation phase. A patient who observes $a=\varnothing$, forms the belief

$$
\left(\frac{1}{\sum_{q^{\prime}=3}^{n} \pi_{q^{\prime}}}\right) \sum_{q=3}^{n} \pi_{q} F^{q} .
$$


Using Lemma 4 and the fact that $\tau \leq \bar{s}$, we deduce (by analogous reasoning to that in the proof of Proposition 3) that it is sequentially rational for the doctor and patient to play the PKBE strategies in the consultation phase. Finally, we may assume that a patient who observes the off-equilibrium advertisement $a \in\{2, \ldots, n\}$ forms the belief $F^{n}$. Since $K<R$ by condition (12), these beliefs satisfy the IC. Given these patient beliefs, the PKBE strategies are sequentially rational, since $\tau \leq s_{n}$ can be deduced from Lemma 4 and the fact that $\tau \leq \bar{s}$.

We next verify that the drug firm's strategy is optimal. By familiar reasoning, each type of drug firm will either choose $a=1$ or $a=\varnothing$. We must show that types $q \in\{1,2\}$ weakly prefer $a=1$ and all other types weakly prefer $a=\varnothing$.

A type $q=1$ firm weakly prefers $a=1 \mathrm{iff}$

$$
K \leq\left[F^{1}(\tau)-\left(1-\sigma_{12}^{*}\right) F^{1}\left(\bar{s}_{12}\right)\right] R
$$

which is condition (12). A type $q=2$ firm weakly prefers $a=1$ iff

$$
\chi \phi \leq\left[F^{2}(\tau)-\left(1-\sigma_{12}^{*}\right) F^{2}\left(\bar{s}_{12}\right)\right] R-K=\bar{x}_{12} .
$$

Similarly, a type $q>2$ firm weakly prefers $a=\varnothing$ iff

$$
\chi \phi \geq\left[F^{q}(\tau)-\left(1-\sigma_{12}^{*}\right) F^{q}\left(\bar{s}_{12}\right)\right] R-K .
$$

Thus, the equilibrium exists iff $\underline{x}_{12} \leq \chi \phi \leq \bar{x}_{12}$.

Proof of Corollary 2. Condition (13) is equivalent to $\underline{x}_{12}>0$. 